This document is confidential and is proprietary to the American Chemical Society and its authors. Do not copy or disclose without written permission. If you have received this item in error, notify the sender and delete all copies.

\title{
Deposition, Characterization, and Thin-Film-Based Chemical Sensing of Ultra-Long Chemically Synthesized Graphene Nanoribbons
}

\begin{tabular}{|r|l|}
\hline Journal: & Journal of the American Chemical Society \\
\hline Manuscript ID: & ja-2014-02764d.R1 \\
\hline Manuscript Type: & Communication \\
\hline Domplete List of Authors: & $\begin{array}{l}\text { Abbas, Ahmad; Univesirty of Southern California, Department of Electrical } \\
\text { Engineering; King Abdulaziz University, Department of Electrical } \\
\text { Engineering } \\
\text { Liu, Gang; Univesirty of Southern California, Department of Electrical } \\
\text { Engineering; UCLA, Chemistry and Biochemistry } \\
\text { Narita, Akimitsu; Max-Planck-Institute for Polymer Research, } \\
\text { Orosco, Manuel; Univesirty of Southern California, Alfred E. Mann Institute } \\
\text { for Biomedical Engineering } \\
\text { Feng, Xinliang; Max-Planck Institute for Polymer Research, Synthetic } \\
\text { Chemistry } \\
\text { Müllen, Klaus; Max-Planck-Institute for Polymer Research, } \\
\text { Zhou, Chongwu; University of Southern California, Dept. of Electrical } \\
\text { Engineering - Electrophysics }\end{array}$ \\
\hline \hline
\end{tabular}

\section{SCHOLARONE ${ }^{\text {M }}$ \\ Manuscripts}


Theoretical predictions of quantum confinement in a graphene lattice have driven lots of research to verify that such confinement can be experimentally realized to create an energy bandgap in graphene. ${ }^{1,2}$ The creation of a bandgap in graphene is essential for the utilization of graphene in digital integrated circuits so that switching off the channel can be realized. Recently, lots of efforts were put into generating GNRs with various widths and lengths using lithographical ${ }^{3-}$ ${ }^{5}$, chemical ${ }^{6-8}$ and various other techniques ${ }^{9-17}$. However, patterning graphene using top-down approaches create GNRs with rough edges which can degrade the carrier transport. ${ }^{18}$ Accordingly, the inability to create GNRs with specific edge structures results in significant sample-tosample variations and disagreement with theoretical predictions on electronic properties. ${ }^{1}$

In order to overcome such problems, various bottom-up chemical methods were developed to control the

\begin{abstract}
Bottom-up synthesis of graphene nanoribbons tronic and sensing applications due to their defined edge structure and high uniformity. Recently, structurally perfect using various chemical synthesis techniques. Nonetheless, issues like GNR deposition, characterization, electronic properties and applications are not fully explored. Here we
report optimized conditions for deposition, characterization and device fabrication of individual and thin films of ultralong chemically synthesized GNRs. Moreover, we have
demonstrated exceptional $\mathrm{NO}_{2}$ gas sensitivity of the GNR film devices down to parts per billion (ppb) levels. The results lay down the foundation for using chemically synthesized GNRs for future electronic and sensing applications.
\end{abstract}

width and edge structure of such GNRs with high uniformity. ${ }^{19-27}$ These methods allow very controllable tuning of the electronic edge structure of GNRs with the possibility of large scale synthesis which is desirable for future electronic and sensing applications of GNRs. Moreover, the latest advancement in chemical synthesis allowed the creation of ultra-long (> $200 \mathrm{~nm}$ ) GNRs which are even processable in liquid-phase, thus facilitating the utilization of such GNRs in various applications. ${ }^{28}$ Nevertheless, some challenges such as GNR deposition (i.e. both isolated individual GNRs and thinfilms), characterization and electronic device measurements are not fully explored.

Here we report optimized conditions for deposition and visualization of individual bottom-up chemically synthesized GNRs and films on $\mathrm{Si} / \mathrm{SiO}_{2}$ substrates, and their applications as devices and chemical sensors. This is in contrast to the previous report, where deposition of GNRs was performed only on conductive substrates, ${ }^{28}$ and could not be directly applied for device studies. Other works, reporting electronic investigation of individual GNRs, employed topdown fabricated GNRs, ${ }^{6-8}$ which might have significantly been affected by nonuniform width and undefined edge structures. We have studied different annealing conditions and their effect on GNR thin-film devices using attenuatedtotal-reflectance Fourier-transform infrared spectroscopy (ATR-FTIR), electrical measurements and Raman spectroscopy. Additionally, we have demonstrated an application of the GNR thin-film devices by measuring the sensitivity of such GNRs to $\mathrm{NO}_{2}$ gas and observed ppb level sensitivity, highlighting the potential for using chemically synthesized GNRs for cost-conscious and scalable sensing applications.

Results and Discussions: 
Figure ra shows the structure of the GNRs used in this study which were synthesized using recently reported methods. $^{23,} 28$ These GNRs have cove-type edge structures with width dimensions of 1.1 and $0.7 \mathrm{~nm}$ (Figure 1a). GNR 1a and $\mathbf{1 b}$ bear dodecyl and tert-butyl ( $t$-Bu) groups, respective$l y$, at the edges, which contribute to their enhanced processability. Additionally, based on the weight-average molecular weight of corresponding polyphenylene precursors, the average length of such GNRs can be estimated to be 500 and $\sim 390 \mathrm{~nm}$ for GNR $\mathbf{1 a}$ and $\mathbf{1 b}$, respectively. ${ }^{23,}{ }^{28}$ Dispersions of GNRs in solvents are usually prepared by sonicating GNR powder in the solvent, followed by centrifugation (Note sı). Figure si shows the ultraviolet-visible (UV-vis) absorption spectrum of GNR $\mathbf{1 a}$ and $\mathbf{1 b}$ dissolved in various solvents. Moreover, the optical bandgap of the GNRs can be extrapolated from Figure si which yields a value of $\sim 1.84 \mathrm{eV}$ comparable to theoretical predictions and other reports. ${ }^{28,29}$ a

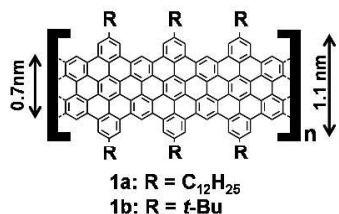

c

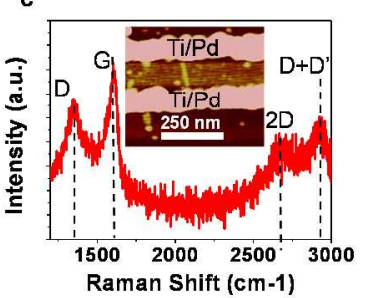

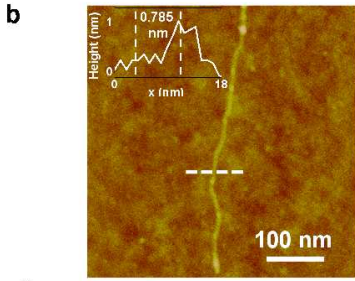

d

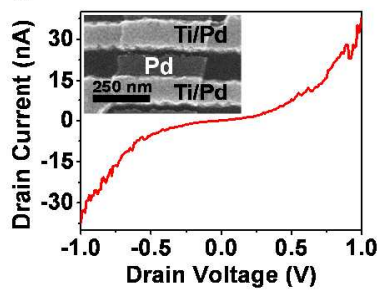

Figure 1. a) Chemical structure of GNRs 1a and 1b. b) AFM tapping mode height image revealing synthesized GNR length $>500 \mathrm{~nm}$. Inset showing a height profile of the GNR (at the dashed line) revealing a height of $0.785 \mathrm{~nm}$. c) Raman spectrum of an individual GNR revealing D, G, 2D and D+D' bands. Inset shows an individual GNR between two $\mathrm{Ti} / \mathrm{Pd}$ electrodes. d) Current vs. drain voltage $\left(I-V_{d}\right)$ characteristic of an individual GNR device after metal angle deposition with a channel length of $\sim 20 \mathrm{~nm}$. Inset showing an SEM

Because the GNRs are long and have extended aromatic structures, strong interaction between GNRs cause their aggregation in the solvent, which can be visually observed as partial precipitation in the dispersions in solvents such as chlorobenzene (CB) and tetrahydrofuran (THF), depending on the concentration and duration after preparation. In spite of the successful synthesis of GNR $\mathbf{1 a}$ and $\mathbf{1 b}$, deposition and visualization of such individual GNR on various substrates has not been previously achieved. In order to image of a $20 \mathrm{~nm}$ gap between Ti/Pd and angle-deposited $\mathrm{Pd}$.

wafer for 24 hours (Note s1). CHP has a boiling point of $\sim 290$ ${ }^{\square} \mathrm{C}$ in atmospheric pressure, and we have observed that GNR dispersions in CHP are very stable with little visible precipitation for the time frame of ten days, in sharp contrast to $\mathrm{CB}$ and THF. Figure $1 \mathrm{~b}$ shows a typical atomic force microscope (AFM) image of a GNR with length $>500 \mathrm{~nm}$ and height of $\sim 0.78 \mathrm{~nm}$ on the functionalized $\mathrm{SiO}_{2}$ surface. Considering the error corresponding to AFM tip radius (i.e. $\sim 2 \mathrm{~nm}$, see Note s1), the observed width of $\sim 5.0$ to $6.9 \mathrm{~nm}$ is consistent with the expected value for a single GNR ra including the alkyl chains $(\sim 3.8 \mathrm{~nm})$. Additionally, most GNRs observed by AFM were 300 to $500 \mathrm{~nm}$ long (Figure s2). In order to locate individual GNRs for recording a Raman spectrum, the measurement was done by patterning two $\mathrm{Ti} / \mathrm{Pd}$ electrodes on an individual GNR (deposited using CHP as a solvent) using electron beam lithography (EBL) and subsequently focusing the laser on the located GNR (Figure 1c). The spectrum shows clear $D, G, 2 \mathrm{D}$ and $\mathrm{D}+\mathrm{D}$ ' peaks, which is consistent with GNR thin-films (Figure 2d) and previously reported spectrum measured on a powder sample of GNR 1a. ${ }^{21,28}$ Electrical measurements on an individual GNR with a channel length of $\sim 100 \mathrm{~nm}$ (Figure $1 \mathrm{c}$ inset) revealed little current conduction in the GNR. Subsequently, we performed metal angle deposition to shorten the channel length to $\sim 20 \mathrm{~nm}$ (Figure id inset) and observed current conduction in the GNR (Figure 1d) which confirms the conductivity of GNR 1a (Note s2).

We have fabricated GNR thin-film devices by dropcasting the dispersion of GNR a in THF or CB as demonstrated in Figure 2a. First, $1 \mathrm{~nm} \mathrm{Ti} / 50 \mathrm{~nm}$ Au electrodes are patterned on top of $\mathrm{P}^{++} \mathrm{Si} / 300 \mathrm{~nm} \mathrm{SiO}_{2}$ substrate (Figure $2 \mathrm{a}$, b). Then GNR dispersion (Note si) is dropped on top of the substrate while heating the substrate to $120{ }^{\square} \mathrm{C}$ to evaporate the solvent. Here, we used THF or CB as the solvent because they can vaporize rapidly and leave a GNR film behind, whilst CHP has a high boiling point and is suitable for incubation to get individual GNRs. Afterwards, the substrate is annealed in $\mathrm{H}_{2} / \mathrm{Ar}$ gases (Note s1). Figure $2 \mathrm{c}$ shows a scanning electron microscope (SEM) image of a GNR film between two Ti/Au electrodes for device study. Moreover, deposition of the GNRs was confirmed by Raman analysis (Figure 2 d). ${ }^{19,21,28}$

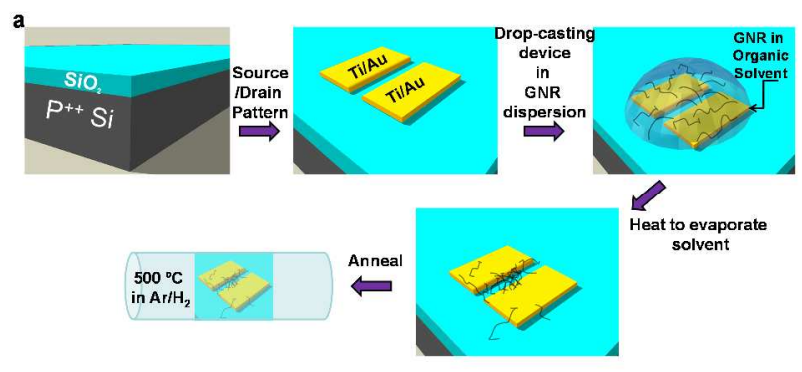
image and characterize individual GNRs on a substrate of choice, aggregation of GNRs in the dispersion should be minimized while adhesion to the substrate must be promoted by the proper surface functionalization. Additionally, it is preferable to have a solvent with a high boiling point so that long incubation times are possible. Based on those requirements, we functionalize the surface of a $\mathrm{Si} / \mathrm{SiO}_{2}$ wafer with dodecyltriethoxysilane while using 1-cyclohexyl-2-pyrrolidone (CHP) as a solvent for GNR incubation on the functionalized

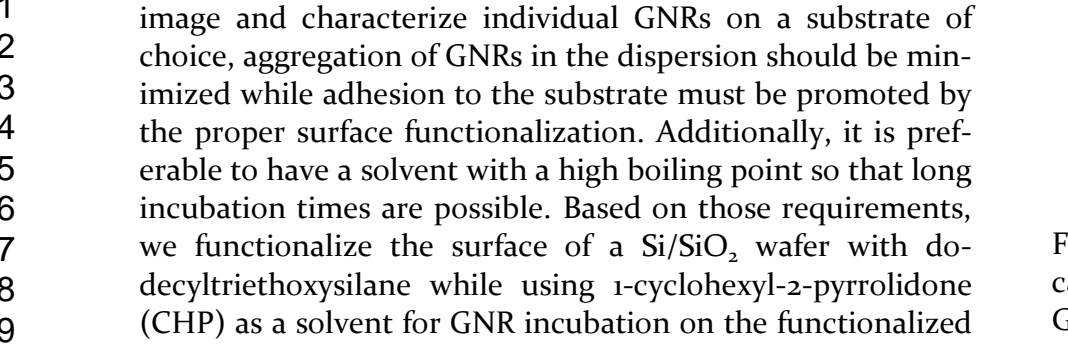

b

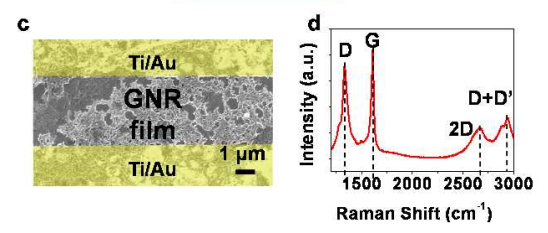

igure 2. a) Scheme of GNR film device fabrication. b) Optical image of the fabricated devices. c) SEM image of a typical GNR film device with GNR films between two (falsely col- 
ored) $\mathrm{Ti} / \mathrm{Au}$ electrodes. d) Raman spectrum of GNR films showing $\mathrm{D}, \mathrm{G}, 2 \mathrm{D}$ and $\mathrm{D}+\mathrm{D}$ ' bands in agreement with single GNR Raman spectrum.

The annealing step after drop-casting the GNR is crucial to enhance the conductivity of GNR film devices. The motivation behind annealing the GNR films was to cut off the insulating alkyl chains from the GNR edges to reduce ribbon-to-ribbon junction resistance without affecting the basal plane. ${ }^{30}$ In order to characterize the annealing effect on the GNR film, ATR-FTIR measurements were carried out on GNR films at different annealing temperatures (Figure 3 a, $\mathrm{b}$ and Figure s4). The spectra were normalized to the peak from conjugated $\mathrm{C}-\mathrm{C}$ at $\sim 1600 \mathrm{~cm}^{-1}$. The clear decrease of the alkyl C-H peaks (i.e. $\sim 1380,1470$ and $2850-2925 \mathrm{~cm}^{-1}$ ) relative to the conjugated $\mathrm{C}-\mathrm{C}$ peak after annealing at 400 and 500 $C$ (Figure $3 a$ and b) suggests the partial removal of alkyl chains from the GNR edges at such conditions. Additionally, the disappearance of the peak at $\sim 720 \mathrm{~cm}^{-1}$, which is also a characteristic peak for alkyl chains, further confirms the removal of alkyl chains from GNR edges (Figure s4). ${ }^{28}$ On the other hand, the peak at $\sim 863 \mathrm{~cm}^{-1}$, which corresponds to $\mathrm{C}-\mathrm{H}$ bonds at the cove position of GNR, ${ }^{28}$ is maintained, which suggests minimal damage to the GNR basal plane after thermal treatment. Moreover, Raman spectroscopy of GNR films after different annealing conditions was carried out to examine the effect of annealing on GNR quality, which revealed no apparent extra defects induced by the thermal treatment (Figure s3). Figure $3 \mathrm{c}-\mathrm{d}$, show the current vs. drain voltage $\left(I-V_{d}\right)$ and current vs. gate voltage $\left(I-V_{g s}\right)$ of a GNR film device after annealing at $500{ }^{\square} \mathrm{C}$, respectively. Figure $3 \mathrm{C}$ inset shows $I-V_{d}$ for a thin-film device before (black curve) and after (red curve) annealing at $500 \square \mathrm{C}$, which confirms that annealing can increase the conduction significantly. Although the GNR is semiconducting, the limited p-type current modulation may be attributed to an electric field screening effect in thick (i.e. 30-100 nm) GNR films and relatively high ribbon-to-ribbon junction resistance (Note s2). ${ }^{30,31}$
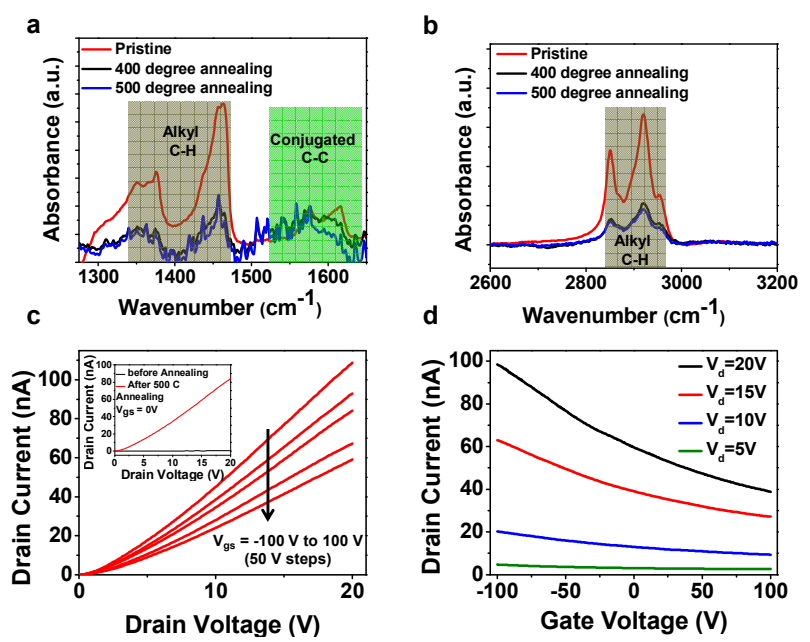

Figure 3. a, b) ATR-FTIR normalized spectra of pristine, 400 and $500 \square$ annealed GNR films showing relative intensity decrease in peaks associated with alkyl $\mathrm{C}-\mathrm{H}$ bonds for annealed samples. c) current vs. drain voltage $\left(I-V_{d}\right)$ of a typical GNR film device under different gate voltages $\left(V_{\mathrm{gs}}\right)$. d) current vs. gate voltage $\left(\mathrm{I}-\mathrm{V}_{\mathrm{gs}}\right)$ of the same device under dif- ferent drain voltages $\left(V_{d}\right)$. Inset shows the $I-V_{d}$ of the device before and after annealing at $500{ }^{\square}$.

In order to demonstrate the applicability of GNR film devices, we used a GNR film device as a $\mathrm{NO}_{2}$ gas sensor. High sensitivity to $\mathrm{NO}_{2}$ is very crucial for human health as relatively low concentrations (i.e. o.2 part per million (ppm)) can cause respiratory irritation. ${ }^{32}$ For sensor measurements, we used $\mathrm{Ar}$ as a dilution gas for different $\mathrm{NO}_{2}$ concentrations and exposed the device to different concentrations while monitoring the relative conductance $\left(G / G_{0}\right)$ as a function of time; where $G$ is the absolute conductance of the device at any given time and $G_{o}$ is the initial conductance of the device before sensing. After exposing the device to a certain $\mathrm{NO}_{2}$ concentration (green arrow), $\mathrm{G} / \mathrm{G}_{\mathrm{o}}$ increases by electron extraction from $\mathrm{NO}_{2}$ molecules (Figure 4a). Then, the sensing chamber is flushed with $\mathrm{Ar}$ to remove $\mathrm{NO}_{2}$ from the chamber (red arrow) and recover $G / G_{0}$ before introducing the new concentration in the chamber. The conductance change of a device $\Delta \mathrm{G} / \mathrm{G}_{\mathrm{o}}$ is defined as: $\Delta \mathrm{G} / \mathrm{G}_{0}=\left(\mathrm{G}-\mathrm{G}_{\mathrm{o}}\right) / \mathrm{G}_{\mathrm{o}}$ and is considered a metric for device sensitivity for a certain concentration. The dependence of $\Delta \mathrm{G} / \mathrm{G}_{\mathrm{o}}$ on the $\mathrm{NO}_{2}$ concentration is plotted in Figure $4 \mathrm{~b}$ and $\mathrm{c}$. The fitted Langmuir Isotherm indicates charge transfer as the sensing mechanism for GNR film devices. We note that the data point for $200 \mathrm{ppb} \mathrm{NO}_{2}$ exposure is an outlier compared to the rest of the data, and the reason may be related to uncontrolled temporal perturbation to the device. Our GNR film sensors exhibit high sensitivities comparing favorably to other graphene-based $\mathrm{NO}_{2}$ sensors ${ }^{33-35}$. For example, Novoselov et al. ${ }^{33}$ and Kim et al. ${ }^{35}$ reported $\Delta G / G_{0}$ of $\sim 4 \%$ and $0.7 \%$ for pristine graphene and ozone treated graphene devices, respectively, exposed to $\mathrm{NO}_{2}$ concentration of $1 \mathrm{ppm}$. On the other hand, we observed $\Delta \mathrm{G} / \mathrm{G}_{\mathrm{o}}$ of $\sim 5.6 \%$ for $\mathrm{NO}_{2}$ concentration of $50 \mathrm{ppb}$ (Note s3).

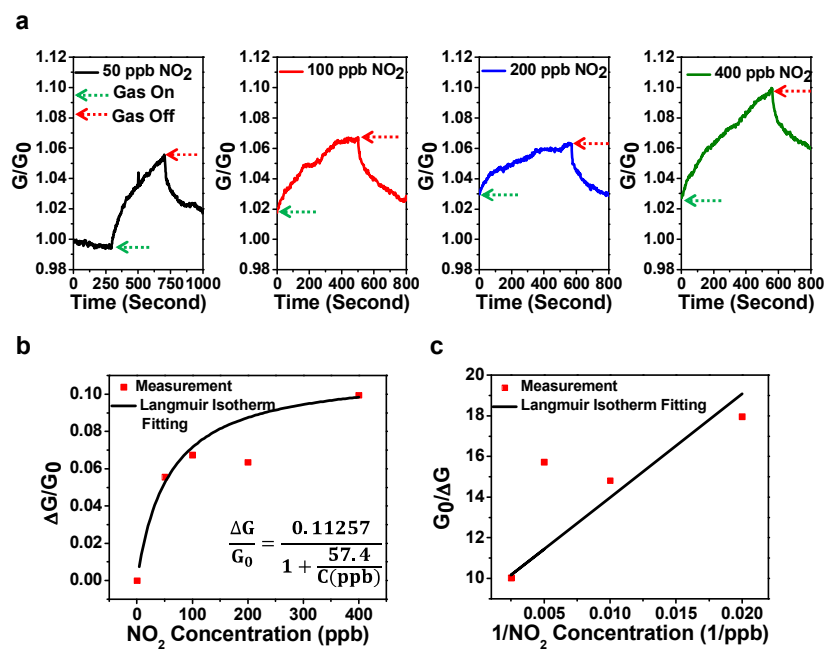

Figure 4. $\mathrm{NO}_{2}$ gas sensing experiment using a GNR film device. a) Time domain normalized conductance $\left(G / G_{0}\right)$ of the GNR film device whilst introducing different concentrations of $\mathrm{NO}_{2}$. Green arrows correspond to the device being exposed to a certain concentration of $\mathrm{NO}_{2}$ while red arrows correspond to the device being flushed with Ar only. b) Measured conductance change $\left(\Delta G / G_{0}\right)$ of the device in (a) as a function of $\mathrm{NO}_{2}$ concentration (red squares) and the corresponding Langmuir isotherm curve fitting (black line). Langmuir isotherm fitted equation is written in bottom right 
corner. c) Inverse conductance change $\left(G_{o} / \Delta G\right)$ vs. inverse $\mathrm{NO}_{2}$ concentration showing a linear relation that further confirms Langmuir isotherm fitting.

\section{Conclusion:}

In summary, a method was developed to deposit individual GNRs on $\mathrm{SiO}_{2}$ using $\mathrm{CHP}$ as a solvent and dodecyltriethoxysilane for surface functionalization. AFM of individual deposited GNRs revealed GNRs with a length of $>500 \mathrm{~nm}$ and a thickness of $\sim 0.78 \mathrm{~nm}$. Additionally, Raman spectroscopy of individual deposited GNRs showed characteristic D, G, 2D, and $\mathrm{D}+\mathrm{D}^{\prime}$ peaks and electrical measurements on an individual GNR confirmed the conductivity of such GNRs. Moreover, GNR film devices were fabricated and ATR-FTIR confirmed the partial removal of alkyl chains from the GNR edges upon annealing the devices at 400 and $500 \quad \mathrm{C}$ which was further supported by the significant conductance increase of GNR film devices. Furthermore, we have demonstrated the exceptional $\mathrm{NO}_{2}$ sensing performance of the GNR film device. The GNR film based sensor is sensitive to low $\mathrm{NO}_{2}$ concentrations down to $50 \mathrm{ppb}$.

\section{ASSOCIATED CONTENT}

\section{Supporting Information}

UV-vis absorption of GNR dispersions (Figure s1), AFM images of GNRs (Figure s2), Raman spectrum of GNR films under different annealing temperatures (Figure s3), more ATRFTIR spectra (Figure s4), Experimental methods (Note s1), Discussion about GNR device study (Note s2), Discussion about GNR film sensing (Note s3). This information is available free of charge via the Internet at http://pubs.acs.org/.

\section{AUTHOR INFORMATION}

\section{Corresponding Author}

Chongwu Zhou

Email: chongwuz@usc.edu

\section{Author Contributions}

$\S$ These authors contributed equally.

Notes

The authors declare no competing financial interests.

\section{ACKNOWLEDGMENT}

We acknowledge the office of Naval Research, European Research Council grant on NANOGRAPH, DFG Priority Program SPP 1459, and European Union Projects UPGRADE, GENIUS, and MoQuaS for financial support.

\section{REFERENCES}

1. Son, Y.-W.; Cohen, M. L.; Louie, S. G., Phys. Rev. Lett. 2006, 97, 216803.

2. Han, M. Y.; Ã-zyilmaz, B.; Zhang, Y.; Kim, P., Phys. Rev. Lett. 2007, 98, 206805.

3. Liang, X.; Wi, S., ACS Nano 2012, 6, 9700-9710.

4. Abbas, A. N.; Liu, G.; Liu, B.; Zhang, L.; Liu, H.; Ohlberg, D.; Wu, W.; Zhou, C., ACS Nano 2014, 8, 1538-1546.

5. Son, J. G.; Son, M.; Moon, K.-J.; Lee, B. H.; Myoung, J.-M.; Strano, M. S.; Ham, M.-H.; Ross, C. A., Adv. Mater. 2013, 25, 47234728.
6. Wang, X.; Dai, H., Nat. Chem. 2o10, 2, 661-665.

7. Wang, X.; Ouyang, Y.; Li, X.; Wang, H.; Guo, J.; Dai, H., Phys. Rev. Lett. 2008, 100, 206803.

8. Li, X.; Wang, X.; Zhang, L.; Lee, S.; Dai, H., Science 2oo8, 319, 1229-1232.

9. Bai, J.; Duan, X.; Huang, Y., Nano. Lett. 2009, 9, 20832087.

10. Abramova, V.; Slesarev, A. S.; Tour, J. M., ACS Nano 2013, 7, 6894-6898.

11. Kato, T.; Hatakeyama, R., Nat. Nanotechnol. 2012, 7, 651656.

12. Sokolov, A. N.; Yap, F. L.; Liu, N.; Kim, K.; Ci, L.; Johnson, O. B.; Wang, H.; Vosgueritchian, M.; Koh, A. L.; Chen, J.; Park, J.; Bao, Z., Nat. Commun. 2013, 4, No. 2402.

13. Pan, Z.; Liu, N.; Fu, L.; Liu, Z., J. Am. Chem. Soc. 2011, 133, 17578-17581.

14. Solís-Fernández, P.; Yoshida, K.; Ogawa, Y.; Tsuji, M.; Ago, H., Adv. Mater. 2013, 25, 6562-6568.

15. Wei, D.; Xie, L.; Lee, K. K.; Hu, Z.; Tan, S.; Chen, W.; Sow, C. H.; Chen, K.; Liu, Y.; Wee, A. T. S., Nat. Commun. 2o13, 4, 1374.

16. Xie, L.; Wang, H.; Jin, C.; Wang, X.; Jiao, L.; Suenaga, K.; Dai, H., J. Am. Chem. Soc. 2011, 133, 10394-10397.

17. Jiao, L.; Zhang, L.; Wang, X.; Diankov, G.; Dai, H., Nature 2009, 458, 877-88o.

18. Pan, M.; Girao, E. C.; Jia, X.; Bhaviripudi, S.; Li, Q.; Kong, J.; Meunier, V.; Dresselhaus, M. S., Nano. Lett. 2012, 12, 1928-1933.

19. Cai, J.; Ruffieux, P.; Jaafar, R.; Bieri, M.; Braun, T.; Blankenburg, S.; Muoth, M.; Seitsonen, A. P.; Saleh, M.; Feng, X. ; Müllen, K.; Fasel, R., Nature 2010, 466, 470-473.

20. Ruffieux, P.; Cai, J.; Plumb, N. C.; Patthey, L.; Prezzi, D.; Ferretti, A.; Molinari, E.; Feng, X.; Müllen, K.; Pignedoli, C. A.; Fasel, R., ACS Nano 2012, 6, 6930-6935.

21. Schwab, M. G.; Narita, A.; Hernandez, Y.; Balandina, T.; Mali, K. S.; De Feyter, S.; Feng, X.; Müllen, K., J. Am. Chem. Soc. 2012, 134, 18169-18172.

22. Koch, M.; Ample, F.; Joachim, C.; Grill, L., Nat. Nanotechnol. 2012, 7, 713-717.

23. Tan, Y.-Z.; Yang, B.; Parvez, K.; Narita, A.; Osella, S.; Beljonne, D.; Feng, X.; Müllen, K., Nat Commun. 2013, 4, No. 2646.

24. Jensen, S. r. A.; Ulbricht, R.; Narita, A.; Feng, X.; Müllen, K.; Hertel, T.; Turchinovich, D.; Bonn, M., Nano Lett. 2013, 13, 59255930.

25. Kim, K. T.; Jung, J. W.; Jo, W. H., Carbon. 2013, 63, 202209.

26. Kim, K. T.; Lee, J. W.; Jo, W. H., Macromol. Chem. Phys. 2013, 214, 2768-2773.

27. Vo, T. H.; Shekhirev, M.; Kunkel, D. A.; Morton, M. D.; Berglund, E.; Kong, L.; Wilson, P. M.; Dowben, P. A.; Enders, A.; Sinitskii, A., Nat Commun. 2014, 5, No. 3189.

28. Narita, A.; Feng, X.; Hernandez, Y.; Jensen, S. r. A.; Bonn, M.; Yang, H.; Verzhbitskiy, I. A.; Casiraghi, C.; Hansen, M. R.; Koch, A. H. R. ; Fytas, G.; Ivasenko, O.; Li, B.; Mali, K. S.; Balandina, T.; Mahesh, S.; De Feyter, S.; Müllen, K., Nat. Chem. 2o14, 6, 126-132.

29. Osella, S.; Narita, A.; Schwab, M. G.; Hernandez, Y.; Feng, X.; Müllen, K.; Beljonne, D., ACS Nano 2012, 6, 5539-5548.

30. Do, J.-W.; Estrada, D.; Xie, X.; Chang, N. N.; Mallek, J.; Girolami, G. S.; Rogers, J. A.; Pop, E.; Lyding, J. W., Nano Lett. 2013, 13, 5844-5850.

31. Sangwan, V. K.; Ortiz, R. P.; Alaboson, J. M. P.; Emery, J. D.; Bedzyk, M. J.; Lauhon, L. J.; Marks, T. J.; Hersam, M. C., ACS Nano 2012, 6, 7480-7488.

32. ACGIH, Threshold Limit Values (TLVs) and Biological Exposure Indices (BEIs) - Nitrogen Dioxide. 2012.

33. Schedin, F.; Geim, A. K.; Morozov, S. V.; Hill, E. W.; Blake, P.; Katsnelson, M. I.; Novoselov, K. S., Nat. Mater. 2007, 6, 652-655.

34. Pearce, R.; Iakimov, T.; Andersson, M.; Hultman, L.; Spetz, A. L.; Yakimova, R., Sens. Actuators, B 2011, 155, 451-455.

35. Chung, M. G.; Kim, D. H.; Lee, H. M.; Kim, T.; Choi, J. H.; Seo, D. k.; Yoo, J.-B.; Hong, S.-H.; Kang, T. J.; Kim, Y. H., Sens. Actuators, $B$ 2012, 166-167, 172-176. 


1
2
3
4
5
6
7
8
9
10
11
12
13
14
15
16
17
18
19
20
21
22
23
24
25
26
27
28
29
30
31
32
33
34
35
36
37
38
39
40
41
42
43
44
45
46
47
48
49
50
51
52
53
54
55
56
57
58
60

SYNOPSIS TOC
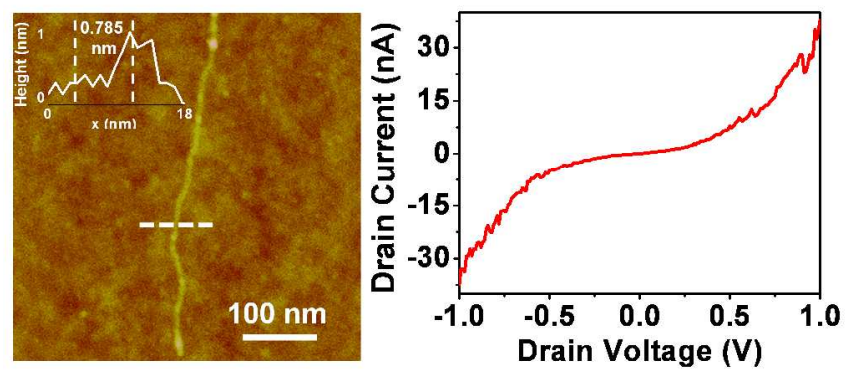

13

14

15

16

17

18

20

21

22

23

24

26

27

28

30

31

32

33

34

35

36

37

38

39

40

41

42

43

44

45

47

48

49

50

51

52

53

54

55

56

57 